\title{
Cloning, Expression, Monoclonal Antibody Preparation of Human Gene NBEAL 1 and Its Application in Targeted Imaging of Mouse Glioma
}

\author{
Chenchen Bao ${ }^{1,2}$, Hao Yang ${ }^{2}$, Ping Sheng ${ }^{1}$, Juxiang Chen ${ }^{1}$, Hua Song ${ }^{2}$, Xuehua Ding ${ }^{1}$, Bin Liu², YichengLu ${ }^{1}$, \\ Guohan $\mathrm{Hu}^{1 *}$, Daxiang Cui ${ }^{*}$
}

\begin{abstract}
${ }^{1}$ Department of Neurosurgery, Changzheng Hospital, Second Military Medical University, Shanghai 200003, China ${ }^{2}$ Department of Bio-Nano Science and Engineering, National Key Laboratory of Nano/Micro Fabrication Technology, Key Laboratory for Thin Film and Microfabrication of Ministry of Education, Institute of Micro-Nano Science and Technology, Shanghai Jiao Tong University, 800 Dongchuan Road, Shanghai 200240, China
\end{abstract}

*Corresponding authors. Email: $\underline{\text { dxcui @ sjtu.edu.cn; huguohao6504@sina.com }}$

\begin{abstract}
Human Neurobeachin-like 1(NBEAL1) gene was an important member of BEACH-WD40 domain family, which was confirmed to be overexpressed in I stage glioma. In this study, we extracted total RNAs from U251 cell line, acquired its cDNA sequence by RT-PCR, and cloned part of NBEAL1 cDNA fragments into the vector pGEX-KG. The recombinant expression vector achieved high expression in E.coli BL21 as a GST fusion protein. NBEAL1 recombinant protein was purified by affinity chromatography. Monoclonal antibody was prepared against the recombinant NBEAL1 protein. Its bioactivity was identified by Western Blotting analysis. Anti-NBEAL1 antibody was conjugated with CdTe quantum dots. Resultant anti-NBEAL1 antibody-conjugated nanoprobes were injected into mice via tail vessel. After $12 \mathrm{~h}$, it is clearly observed that prepared nanoprobes located in brain tissues of mice model with glioma by IVIS Imaging system. In conclusion, NBEAL1 protein was successfully expressed and its monoclonal antibody was successfully prepared. Anti-NBEAL1 antibody-conjugated quantum dots may be used to image glioma. These prepared nanoprobes have great potential in early detection of glioma.
\end{abstract}

Keywords: NBEAL1; glioma; clone; expression; monoclonal antibody

Citation: Bao C, et al. Cloning, Expression, Monoclonal Antibody Preparation of Human Gene NBEAL1 and Its Application in Targeted Imaging of Mouse Glioma. Nano Biomed. Eng. 2009, 1(1), 50-56. DOI: 10.5101/nbe.v1i1.p50-56

\section{Introduction}

Gliomas, the most common tumors of the Central Nervous System (CNS), account for $45-50 \%$ of all primary brain tumors and almost $80 \%$ of primary malignant brain tumors, and are considered to be originated from glial cells $[1,2]$. According to features of histological aberrations of gliomas, they are graded into grade I, II, III and IV in the World Health Organization (WHO) classification of tumors of the nervous system in 2002 and 2007 [3-6]. Among gliomas, those without atypical cells, mitoses, endothelial proliferation and necrosis, such as pilocytic astrocytoma(PA), dysembryoplastic neuroepithelial tumor(DNET) and ganglioglioma etc, are classified as WHO grade I. Those possessing only one feature mentioned above are classified as WHO grade II. WHO grade III and IV gliomas are those with two or more mentioned above features and considered to be malignant [6-8]. In general, radiological imaging and histopathology are the most common tools for the diagnosis of gliomas, and the latter is the 
current golden standard for diagnosis and classification of gliomas. However, subjective criteria, tissue sampling error and lack of specific tumor markers remain to be the unsolved challengeable problems for detection of early gliomas [9-11].

Human neurobeachin-like 1(NBEAL1) is a novel gene which was firstly isolated from fetal brain in our cooperative laboratory [12]. Its cDNA is 3858 bp containing an Open Reading Frame (ORF) of 3006 bp encoding a putative 1001 amino-acid protein. This gene is located on human chromosome 2q33-2q34 and encompasses 25 exons. Based on the results of reverse transcription-polymerase chain reaction (RT-PCR), it could be detected in human tissues including brain, kidney, prostate, testis, small intestine, colon and peripheral blood leukocyte, and almost undetectable in heart, placenta, lung, liver, skeletal muscle, pancreas, spleen, thymus and ovary [12]. More important, RT-PCR and Northern Blotting analysis confirmed that this novel gene exhibited higher expression in the biopsies of grade I of glioma, and lower expression in other grades of gliomas, which highly suggests that NBEAL1 gene may be one biomarker closely associated with the grade I of gliomas. However, up to date, no report is closely associated with the function of protein of NBEAL1 in progression of gliomas.

Quantum dots have been subject to intensive investigations due to their unique properties and potential application prospect [13-15]. So far, several methods have been developed to synthesize water-soluble QDs for use in biological relevant studies [16-17]. For example, QDs have been used successfully in cellular imaging [18-19], immunoassays [20], DNA hybridization [21] and optical barcoding [22]. Quantum dots provide a new functional platform for bioanalytical sciences and biomedical engineering. In this study, we constructed the expression vector of NBEAL1 gene, isolated and purified GST-tagged NBEAL1 protein, prepared anti-NBEAL1 monoclonal antibody, and then conjugated with CdTe quantum dots. Resultant nanoprobes were used for targeted imaging of mouse glioma. Our primary results lay foundation for further clinical application.

\section{Materials and Methods}

\subsection{Cloning of Nbeal-1 gene and its expression vec- tor construction}

Total RNAs was isolated from glioma U251 Cell lines (purchased from Institute of Biochemistry and Cell Biology, Shanghai Institute for Biological Sciences, CAS) according to the manufacturer's protocol (ROCHE, Mannheim Germany). The concentration and the purity of the isolated total RNAs were determined by Uv-vis spectrophotometer. The integrity of the RNAs was confirmed by gel electrophoresis. The cDNAs was then synthesized from $2 \mu \mathrm{g}$ of total RNAs using Oligo (dT)18-20 as primers according to the manufacturer's protocol (ROCHE, Mannheim Germany).

The NBEAL1 gene fragment of 328 bp in length was specifically amplified by using cDNAs of NBEAL1 as template. The forward and reverse primers flanking the NBEAL1 were as follows:

NB348F:

5'-CCGGAATTCTAGACTTTGTCGACTC-3' and

NB348R:

5'-CCGCTCGAGTAAGACGTTAACGCG-3'.

$E c o R I$ and $X$ ho $I$ restriction sites were introduced at the 5 'end of NB348F and NB348R primers respectively to facilitate cloning into pGEX-KG vector (a generous gift from Dr KunLiang Guan, Purdue University, USA), and the Nbeall gene fragment was amplified by using PCR kit from TaKaRa Company (Takara, Dalian, China). The $50 \mu \mathrm{L}$ reaction mixture contained $5.0 \mu \mathrm{L}$ $10 \times$ Ex Taq Buffer ( $\mathrm{Mg}^{2+}$ Plus), $10 \mathrm{pM}$ each of forward and reverse primers, $100 \mathrm{ng}$ of Nbeall cDNAs as template and $2.5 \mathrm{U} / \mu \mathrm{L}$ of EX TaqTM Enzyme.

The PCR amplification involved initial denaturation at $94{ }^{\circ} \mathrm{C}$ for $5 \mathrm{~min}$, followed by 35 cycles each consisting of a $30 \mathrm{sec}$ denaturation at $94{ }^{\circ} \mathrm{C}$, a $30 \mathrm{sec}$ annealing at $50{ }^{\circ} \mathrm{C}$, and a $1 \mathrm{~min}$ extension at $72{ }^{\circ} \mathrm{C}$. To facilitate TA cloning, the final extension at $72{ }^{\circ} \mathrm{C}$ was allowed for $7 \mathrm{~min}$. The amplified PCR product was analyzed by $1 \%$ agarose gel electrophoresis. The PCR yields were purified by using TaKaRa DNA Fragment Purification Kit Ver.2.0 (Takara, Dalian, China) before ligated into the TA vector pMDTM 18-T Vector (TaKaRa, Dalian, China), and transformed into DH5a E. coli cells. The cells were plated onto agar plates containing $100 \mu \mathrm{g} / \mathrm{mL}$ ampicillin. Positive recombinant clones of Nbeal1 were selected by colony PCR. Plasmids were isolated and digested with EcoR I and Xho $I$ restriction enzymes. The digested fragments were run on $1.0 \%$ agarose gel, and the Nbeall gene fragment of $348 \mathrm{bp}$ in length was sliced from gel and eluted by gel extraction columns. The collected DNA fragments were ligated between the EcoR $I$ and Xho $I$ sites of pGEX-KG expression vector. The recombinant pGEXKG -Nbeall clones were selected by colony PCR, and confirmed by restriction enzyme digestion.

\subsection{Expression and purification of NBEAL-1 pro- tein}

The recombinant pGEX-KG-NBEAL1 plasmids were sequenced before transforming into E.coli BL21 DE3 cells. The E.coli BL21 DE3 cells containing the pGEX-KG-NBEAL1 plasmid were grown in $2 \times Y T$ medium containing ampicillin $(100 \mu \mathrm{g} / \mathrm{L})$. Typically, $1 \mathrm{~mL}$ of overnight cultured bacteria was added to a $100 \mathrm{~mL}$ medium, and grown up to OD600 $=0.5$ at $37^{\circ} \mathrm{C}$. The target protein expression was then induced by adding IPTG to a final concentration of $0.6 \mathrm{mM}$, and the growth was continued for $4 \mathrm{~h}$. Save $1 \mathrm{~mL}$ aliquot every hour for SDS-PAGE analysis. The rest was 
harvested after $4 \mathrm{~h}$ by centrifugation at $7700 \mathrm{~g}$ for 10 min at $4^{\circ} \mathrm{C}$. GSTrap 4B HiTrap affinity columns (GE Healthcare, Uppsala, Sweden) were used for purification. Harvested cells was immediately suspended with $5 \mathrm{~mL}$ cold binding buffer $(140 \mathrm{mM} \mathrm{NaCl}, 2.7 \mathrm{mM} \mathrm{KCl}$, $10 \mathrm{mM} \mathrm{Na}_{2} \mathrm{HPO}_{4}, 1.7 \mathrm{mM} \mathrm{KH}_{2} \mathrm{PO}_{4}, \mathrm{pH}$ 7.4). Suspended cells were then disrupted with a sonicator on ice in short bursts until it became clear and frothing needed to be avoided in this process. This sample should be centrifuged and/or filtered through a $0.45 \mu \mathrm{m}$ filter before it is applied to the equilibrated column. A syringe is used to pump the sample into the column and the flow rate should be controlled between 0.2 to $1 \mathrm{~mL} / \mathrm{min}$ during sample application. After washing with $5 \mathrm{~mL}$ of binding buffer, the samples were eluted with $5 \mathrm{~mL}$ of elution buffer $(50 \mathrm{mM}$ Tris- $\mathrm{HCl}, 10-20 \mathrm{mM}$ reduced glutathione, $5 \mathrm{mM}$ DTT, pH 8.0). SDS-PAGE analysis was used to confirm the expression analysis of Nbeall protein.

\subsection{Preparation of monoclonal Antibodies}

Monoclonal antibodies were prepared against a purified fusion protein GST-NBEAL1. BALB/c female mice, 4-6 weeks old, were purchased from the Animal Center of Shanghai Jiaotong University. The mice were immunized by intraperitoneal injection with $50 \mu \mathrm{g}$ of purified GST-NBEAL1 which was emulsified with an equal volume of Freund's complete adjuvant. Three further injections were administered using incomplete adjuvant every 2 weeks. Three days after the last injection, the spleen cells of the mice were harvested and fused with the $\mathrm{Sp} 2 / 0$ mouse myeloma cell line. After 10-14 days, the culture supernatants were screened with an ELISA test in which the solid phase was coated with the recombinant GST-NBEAL1 protein $(2 \mu \mathrm{g} / \mathrm{mL})$ used for the immunization and GST protein expressed by pGEX-KG vector $(2 \mu \mathrm{g} / \mathrm{mL})$. In the screening process, the monolcone antibodies to bind with coated
GST-NBEAL1 but not to bind with coated GST protein were selected. By twice limiting dilution, positive colonies were subcloned. Ascitic fluids were harvested from the mice primed with a $0.5 \mathrm{~mL}$ intraperitoneal injection of Pristane and then injected with $10^{6}$ hybridoma cells. The class and subclass of each mAb were determined using a mouse monoclonal antibody isotyping kit (HyCult Biotechnology B.V., Netherlands). The $\mathrm{mAbs}$ were purified from the mouse ascetic fluids using a protein G-Sepharose 4FF (Pharmacia, Uppsala, Sweden) column according to the manufacturer's instructions to remove components which might interfere with the biopanning experiments. The antibody titers were determined by ELISA methods as table 1 .

\subsection{Western Blotting Analysis}

Isolation of proteins was performed by $12 \%$ SDS (sodium dodecyl sulfate)-polyacrylamide gel electrophoresis. After separation, proteins were either stained with Coomassie Brilliant Blue (CBB) reagent or electrotransferred onto PVDF (Polyvinylidene Difluoride)membrane (Immobilon-P, Millipore, Eschborn). Electrotransfer was performed at room temperature for $1 \mathrm{~h}$ with $0.8 \mathrm{~mA} / \mathrm{cm}^{2}$ membrane. The membranes were incubated in a blocking solution $(50 \mathrm{mM}$ Tris, $150 \mathrm{mM}$ $\mathrm{NaCl}, 0.05 \%$ Tween $20(\mathrm{v} / \mathrm{v})$, agitating at $37^{\circ} \mathrm{C}$ for $1 \mathrm{~h}$. The membranes were then incubated with rabbit polyclonal antibodies against GST(Proteintech Group Inc, Chicago, USA) diluted 1:1000, or mouse monoclonal antibody against NBEAL1 diluted 1:2000, in 1\% (v/v) blocking solution, followed by incubation with peroxidase-conjugated secondary antibodies (Proteintech Group Inc, Chicago, USA) for polyclonal and monoclonal antibodies were anti-rabbit IgG (diluted 1:10000) or anti-mouse IgG (diluted 1:7500), respectively. Visualization was performed with Lumi-Light Western Blotting Substrate

Table 1. Titers of Anti-NBEAL1 Monoclonal Antibodies in Ascites Fluid Induced by

Hybridoma Clone Cells by ELISA

\begin{tabular}{|c|c|c|c|c|}
\hline \multirow[b]{2}{*}{ Clone } & \multicolumn{4}{|c|}{ Antibody titer* } \\
\hline & NBEAL1S-OVA ** & RGD(C)-BSA *** & $\mathrm{BSA}^{* *}$ & OVA ** \\
\hline S-200-5 & $1,024,000$ & $1,024,000$ & $<1,000$ & $<1,000$ \\
\hline S-335-5 & 128,000 & 512,000 & $<1,000$ & $<1,000$ \\
\hline
\end{tabular}

*The reciprocal of ascites fluid dilution

The first dilution of ascites fluid was 1:1,000.

*** The antigens were coated on ELISA plate. 
(ROCHE Mannheim Germany) followed by an exposure on X-ray film for $1 \mathrm{~h} \mathrm{[23].}$

\subsection{Preparation of nanoprobes}

CdTe quantum dots were prepared according to our previous papers [19-21] and were conjugated with anti-NBEAL1 monoclonal antibody according to our previous reports [19-21]. Then the resultant nanoprobes were purified by HPLC and kept at $4{ }^{\circ} \mathrm{C}$ for usage.

\subsection{Preparation of nude mice model with glioma and Imaging}

Animal experiments were performed according to Guidelines for Animal Care and Use Committee, Shanghai Jiao Tong University. glioma U251 Cells $\left(5 \times 10^{6}\right)$ were injected into brain tissues of nude mice of age 6-8 weeks. These mice were continued to raise for one week. Then $200 \mu \mathrm{g}$ of prepared nanoprobes was injected into mice via tail vein, continued to breed for $6 \mathrm{~h}$, before those mice were imaged by using IVIS Imaging System.

\section{Results}

\subsection{Cloning and identification of Nbeal-1 gene fragments}

The recombinant pGEX-KG-NBEAL1 vector was confirmed by PCR and restriction enzyme digestion for 1 hour. The PCR products and double enzyme digestion yields were analyzed by $1 \%$ agarose gel electrophoresis; the result is shown in Figure1 and Figure 2, a DNA fragment of 348 bp in length was observed, which corresponded to the size of the aimed fragment. The recombinant pGEX-KGNBEAL1 vector was also sequenced and confirmed that the inserted gene fragment was right. Our results showed that Nbeal-1 gene fragment was successfully cloned into pGEX-KG expression vector.

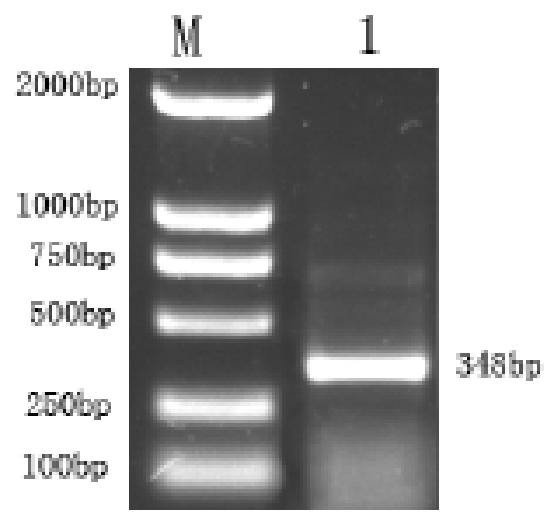

Figure 1. 1\% agarose gel electrophoresis analysis of NBEAL1 gene fragment amplified by PCR Lane M, DNA marker; lane 1, amplified NBEAL1 gene fragment

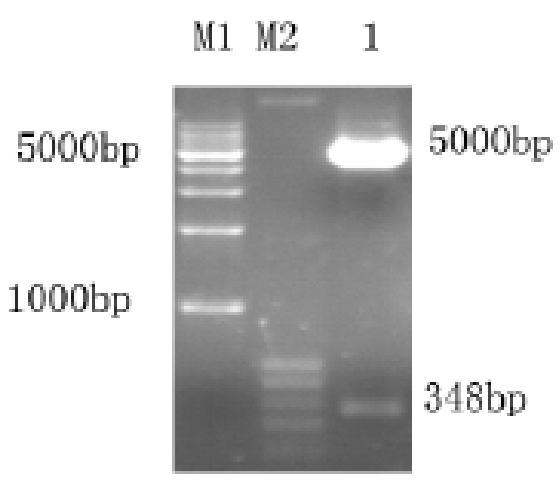

Figure 2. $1 \%$ agarose gel electrophoresis analysis of enzyme digestion products Lane M1, DNA marker 1; lane M2, DNA marker 2; lane 1, after one hour double enzyme digestion, fragments of $5000 \mathrm{bp}$ pGEX-KG and 348 bp NBEAL1 fragment are detected.

\subsection{Expression and purification of pGEX-KG- NBEAL1 vector}

Expression of GST-tagged NBEAL1 gene was confirmed by SDS-PAGE and Western blotting analysis. As shown in Figure3A, a major protein band of about $39 \mathrm{kDa}$ only appeared in the induced cells, and the amounts of expressed protein increased as the induced time increased, lane 7 showed the purified proteins. As shown in Figure3B, these expressed proteins could react with both the anti-GST (polyclonal) and the anti-NBEAL1 (monoclonal) antibodies, highly suggesting that this protein band included the human recombinant NBEAL1 protein, and owned bioactivity.

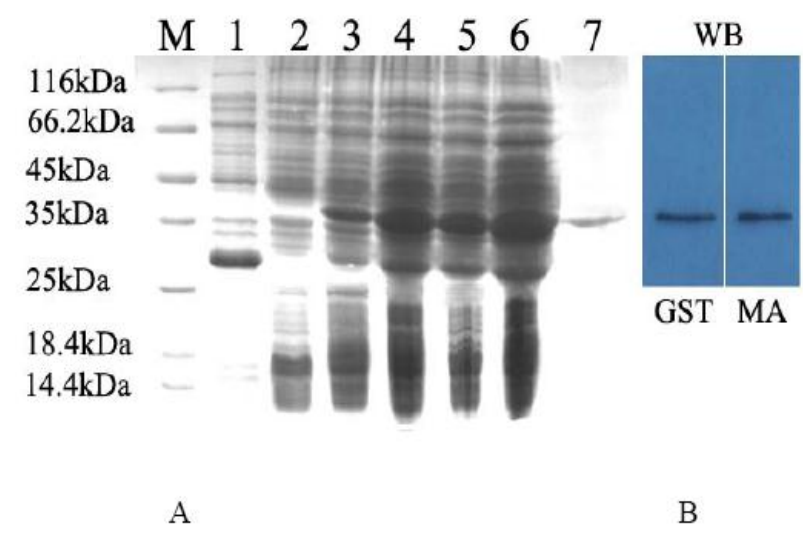

Figure 3. Expression and purification of recombinant protein 


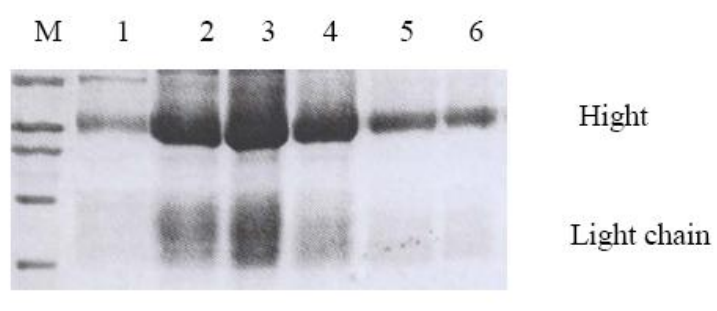

Figure 4. 12\% SDS-PAGE analysis of Monoclonal antibodies M: protein marker; lane 1: control; lane 2-4: unpurified monoclonal antibody; lane 5,6: purified monoclonal antibody

M: protein Marker; 1: IPTG(+)pGEXKG(+)NBEAL(-);2:IPTG(-)pGEX-

KG(+)NBEAL (+);3-6: $\quad$ PTG(+)pGEX$\mathrm{KG}(+)$ NBEAL(+) from $1 \mathrm{~h}$ to $4 \mathrm{~h}$; 7 : purified GST tagged NBEAL1; GST: WB result of Rabbit Anti human GSTT1 MonoClonal antibody; MA: Western Blot result by using mouse anti-human NBEAL1 monoclonal antibody as first antibody; GST: Western blot result by using mouse anti-GST antibody as first antibody.

\subsection{Preparation of monoclonal antibodies against GST-tagged NBEAL1 protein}

As shown in Figure 4, the purified monoclonal antibodies were successfully prepared.

We obtained two monoclonal antibody cell lines. Their bioactivities were identified by ELISA.

\subsection{Imaging mice with glioma by IVIS Imaging System}

As shown in Figure 5A, prepared CdTe quantum dots were $3 \mathrm{~nm}$ or so in diameter. Figure 5B showed the PL intensity of CdTe quantum dots (A), CdTe quantum dots conjugated with Anti-NBEAL-1 antibody (B). The PL peak of prepared nanoprobes exhibit blue shift compared with CdTe quantum dots. As shown in Figure 5C, after prepared nanoprobes were injected into mice for $24 \mathrm{~h}$, nanoprobes can be clearly observed to locate in the brain of mouse, which showed that as-prepared nanoprobes own brain targeted ability.

\section{Discussion}

Glioma is one kind of common brain tumor. Looking for its novel biomarkers helps us to clarify its mechanism in favor of its diagnosis, treatment, and prevention. So far, although some useful biomarkers associated with glioma have been recognized, how to realize its early diagnosis and distinguish their malignant degree is still a great challengeable problem for glioma therapy. Our previous work showed that the transcripts of NBEAL1 were highly expressed in the low-grade of human glioma, for example, grade I , but lower or not expressed in the high-grade gliomas( grade II, III and IV) [12]. In this study, we expressed NBEAL1 proteins and prepared successfully its monoclonal antibody. Then we fully used the advantages of quantum dots [21], conjugated the prepared monoclonal antibody with CdTe quantum dots (QDs). The resultant nanoprobes exhibited fluorescent signals, which successfully realized imaging the glioma in the mouse model, which highly suggest that NBEAL1 protein may be a potential biomarker for glioma, its antibody has potential application in imaging diagnosis of glioma.

So far, NBEAL1 was found to possess the closest similarity with NBEA within the Beige and Chediak-Higashi (BEACH) domain. It also contained a $\mathrm{BEACH}$ domain from amino acide residues 311 to 561 and three WD40 repeats from amino acid residues 737 to 962 [12]. BEACH domain plays important role in vesicle trafficking, membrane dynamics and receptor signaling [24]. It also shares the homologous sequences with other BEACH family members, such as NBEA (57.7\% identity), human FAN (49.6\% identity), human LYST $(48.8 \%$ identity). The high conservation of the BEACH domain of NBEAL1 may suggest that it might have the similar properties as NBEA or the other homologous protein. The latter is crucial for their functions in vesicle trafficking, membrane dynamics and receptor signaling [25]. NBEAL1 has the closest relationship with Neurobeachin (NBEA), which is implicated in the neuromuscular synaptict ransmission [16], protein kinase A binding [27], and which was recently reported as a novel potential plasma cell dyscrasia tumor suppressor gene [28]. NBEAL1 also has a vacuolar targeting motif ILPK which implies its location in lysosomes. So far our results also support this hypothesis. ur study firstly obtained the biologically active peptides of NBEAL1. Unfortunately, though different vectors induce temperatures or IPTG concentration was tried, we failed to express the full length of NBEAL1. It might because of the relatively high content of rare codons in NBEAL1. Through predictive analysis of antigenic index (http://immunax.dfci.harvard.edu/Tools/antigenic.pl), we finally shorten the fragment to $348 \mathrm{bp}$, which contains 6 antigen epitopes, and cloned it into pGEXKG. The biologically activity was proved by Western-Blotting Analysis.

Looking for new glioma biomarkers are of great importance for early glioma diagnosis and therapy because they could be used as tools for diagnosis and prognosis, molecular targets for monitoring tumor initiation and progression as well as therapeutics [29]. For low grade gliomas (grade I and II), there existed several potential biomarkers up to date. For example, Loss of heterozygosity (LOH) on chromosomes $1 \mathrm{p}$ and $19 q$ was reported to be of both diagnostic and prognostic values [4]. Development of low grade astrocytoma is thought to involve TP53 mutation, 

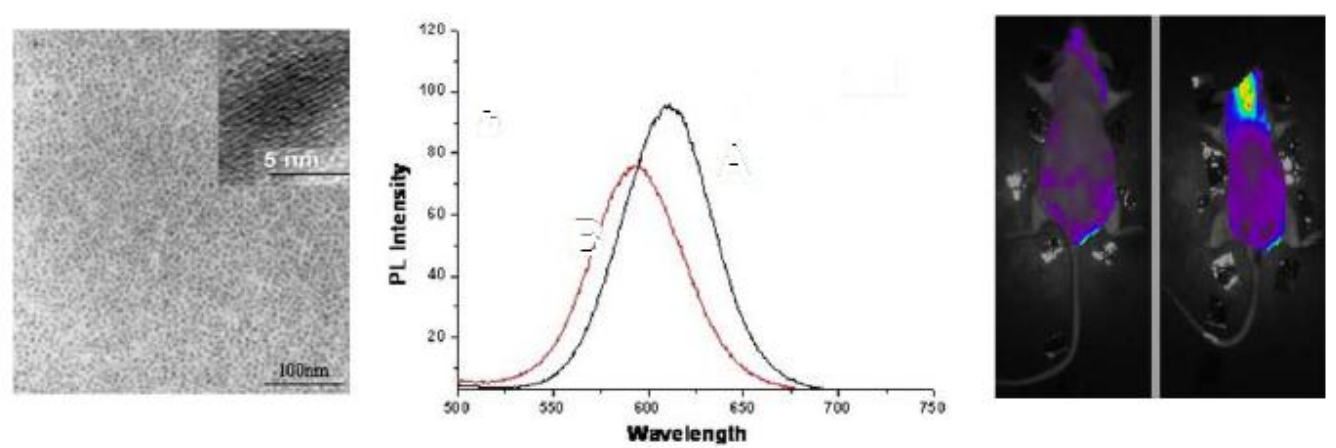

Figure 5. (a) High-resolution transmission electron microscopy images of CdTe quantum dots; (b) PL spectra of as-prepared nanoprobes before and after conjugated with antibody; (c) images of mouse injected with as-prepared nanoprobes for $24 \mathrm{~h}$, control mouse (C1) and test mouse (C2).

over-expression of PDGF (platelet-derived growth factor), FGF (fibroblast growth factor) and their receptors and 22q loss [30]. Moreover, Ki-67/MIB-1, which is reactive against $\mathrm{Ki}-67$ nuclear antigen, is reported to have $1-2 \%$ of labeling index in diffuse grade II astrocytoma and $15-20 \%$ of labeling index in WHO grade IV astrocytoma [31]. To investigate the characteristic expression of NBEAL1 and verify our previous Reverse Transcript-PCR results, we prepared monoclonal antibodies against NBEAL1 and applied them to target and image the glioma. The results are quite promising. Anti-NBEAL1 antibodyconjugated QDs nanoprobes can get through the blood-brain barrier, and enter into brain tissues of mouse loaded with glioma, which highly suggest that the prepared nanoprobes own specificity.

In summary, we successfully prepared NBEAL1 proteins, prepared its monoclonal antibodies. The monoclonal antibody-conjugated CdTe quantum dots can get through blood brain barrier and enter into brain tissues in mice loaded with glioma, which highly suggest that prepared nanoprobes have great potential in application such as early diagnosis of glioma. However, so far we still do not clarify the function of NBEAL1 protein; the as-prepared nanoprobes are still not evaluated according to biosafety standard. All of the works mentioned above will be done in near future. Our primary results lay foundation for further investigating the function of NBEAL1 protein.

\section{Acknowledgments}

This work is supported by China National 973 Project (No.2010CB933901), National 863 Hi-tech Project (Nos. 2007AA022004 and 2005AA001070), National Natural Scientific Fund (No.30672147). The authors thank the Instrumental Analysis Center of Shanghai Jiao Tong University for the Materials Characterization.

\section{References}

1. Schwartzbaum JA, Fisher JL, Aldape KD, Wrensch M. Epidemiology and molecular pathology of glioma. Nat Clin Pract Neuro 2006; 2: 494-503. doi:10.1038/nc pneuro0289

2. Lu C, Shervington A. Chemoresistance in gliomas. Mol Cell Biochem 2008; 312: 71-80. doi:10.1007/s11010008-9722-8

3. Kleihues P, Louis DN, Scheithauer BW, Rorke LB, Reifenberger G, Burger PC, Cavenee WK. The WHO classification of tumors of the nervous system. $J$ Neuropath Exp Neur 2002; 61: 215.

4. Louis DN, Ohgaki H, Wiestler OD, Cavenee WK. WHO classification of tumours of the central nervous system: International Agency for Research on Cancer Lyon, France, 2007.

5. Louis DN, Ohgaki H, Wiestler OD, Cavenee WK. Burger PC, Jouvet A, Scheithauer BW, Kleihues P. The 2007 WHO classification of tumours of the central nervous system. Acta neuropathologica 2007; 114: 97-109

6. Martin-Villalba A, Okuducu AF, von Deimling A. The evolution of our understanding on glioma. Brain Pathology 2008; 18: 455-463. doi:10.1111/j.1750$\underline{3639.2008 .00136 . x}$

7. Nakada M, Nakada S, Demuth T, Tran NL, Hoelzinger DB, Berens ME. Molecular targets of glioma invasion. Cell Mol Life Sci 2007; 64: 458-478. doi:10.1007/s000 18-007-6342-5

8. Grier JT, Batchelor T. Low-grade gliomas in adults. The Oncologist 2006; 11: 681. doi:10.1634/theoncologist.11$\underline{6-681}$

9. McKnight TR, von dem Bussche MH, Vigneron DB, Lu Y, Berger MS, McDermott MW. Histopathological validation of a three-dimensional magnetic resonance spectroscopy index as a predictor of tumor presence. $\mathrm{J} \mathrm{Neu}$ rosurg 2002; 97: 794-802. 이:10.3171/jns.2 002.97.4.0794

10. Galanaud D, Chinot O, Nicoli F, Confort-Gouny S, Le Fur Y, Barri Attarian M, Ranjeva JP, Fuent S, Viout P, Figarella-Branger D. Use of proton magnetic resonance spectroscopy of the brain to differentiate gliomatosis cerebri from low-grade glioma. J Nneurosurg 2003; 98:269276. doi:10.3171/jns.2003.98.2.0269

11. Jeuken JWM, Deimling AV, Wesseling P. Molecular pathogenesis of oligodendroglial tumors. J Neuro-oncol 2004; 70: 161-181. doi:10.1007/s11060-004-2748-1

12. Chen J, Lu Y, Xu J, Huang Y, Cheng H, Hu G, Luo C, Lou M, Cao G, Xie Y, Ying K. Identification and characterization of NBEAL1, a novel human neurobeachin-like 1 protein gene from fetal brain, which is up regulated in glioma. Brain Res Mol Brain Res 2004; 125: 147-155. doi:10.1016/j.molbrainres.2004.02.022 
13. Jeong H, Chang AM and Melloch MR. The Kondo Effect in an Artificial Quantum Dot Molecule. Science 2001; 293: 2221-2223. doi:10.1126/science. 1063182

14. Li XQ, Wu YW, Steel D, Gammon D, Stievater TH, Katzer DS. An all-optical quantum gate in a semiconductor quantum dot. Science 2003; 301: 809-811. doi: $10.1126 /$ science. 1083800

15. Lu W, Ji ZQ, Pfeiffer L, West KW, Rimberg AJ. Realtime detection of electron tunnelling in a quantum dot. Nature 2003; 423: 422-425. doi:10.1038/nature01642

16. You X, He R, Gao F, Shao J, Pan B, Cui D. Preparation and characterization of $\mathrm{CdTe} @ \mathrm{SiO}_{2}$ core/shell luminescent composite nano particles. Acta Chimi Sin 2007; 65:561-565.

17. Kaul Z, Yaguchi T, Kaul SC, Hirano T, Wadhwa R and Taira K. Mortalin imaging in normal and cancer cells with quantum dot immuno-conjugates. Cell Res 2003; 13: 503-507. doi:10.1038/sj.cr.7290194

18. Medintz IL, Uyeda HT, Goldman ER and Mattoussi H. Quantum dot bioconjugates for imaging, labelling and sensing. Nature Mater 2005; 4: 435-446. doi:10.1038/nmat1390

19. Shao J, You X, Gao F, He R, Cui D. Labeling of quantum dots with streptavidin and its bioactivity measurement. Chinese J Anal Chem 2006; 34:1625-1628.

20. He R, You X, Shao J, Gao F, Pan B, Cui D. Core/shell fluorescent magnetic silica-coated composite nanoparticles for bioconjugation. Nanotechnology 2007; 18, 315601. doi:10.1088/0957-4484/18/31/315601

21. Cui D. Pan B. Zhang H, Wu R, Wang J, Asahi T, et al. Self/Assembly of Quantum Dots and Carbon Nanotubes For Ultrasensitive DNA and Antigen Detection. Anal Chem 2008; 80: 7996-8001. doi:10.1021/ac800992m

22. Li Z, Huang P, He R, Lin J, Yang S, Zhang X, et al. Aptamer-conjugated dendrimer-modified quantum dots for cancer cell targeting and imaging. Mater Lett 2010; 64: 375-378. doi:10.1016/j.matlet.2009.11.022

23. Cui D, Gao T, Jin D, Sun T, Tian F, Estrada GG, et al. Characterization of BRCAA1 and its clinical significance. Cancer Epid. Biomarker Prev 2004; 13: 1336-1445.

24. Jogl G, Shen Y, Gebauer D, Li J, Wiegmann K, Kashkar
$\mathrm{H}$, Kroke M, et al. Crystal structure of the BEACH domain reveals an unusual fold and extensive association with a novel PH domain. EMBO J 2002; 21: 4785-4795. doi:10.1093/emboj/cdf502

25. De Lozanne A. The Role of BEACH Proteins in Dictyostelium. Traffic 2003; 4: 6. doi:10.1034/j.16000854.2003.40102.x

26. Su Y, Balice-Gordon RJ, Hess DM, Landsman DS, Minarcik J, Golden J, et al. Neurobeachin is essential for neuromuscular synaptic transmission. J Neurosci 2004; 24: 3627-3636. doi:10.1523/JNEUROSCI.4644-03.2004

27. Wang X, Herberg FW, Laue MM, Wullner C, Hu B, Petrasch-Parwez E, et al. Neurobeachin: A protein kinase A-anchoring, beige/Chediak-higashi protein homolog implicated in neuronal membrane traffic. J Neurosci 2000; 20: 8551-8565.

28. O'Neal J, Gao F, Hassan A, Monahan R, Barrios S, Lee I, et al. Neurobeachin (NBEA) is a target of recurrent interstitial deletions at $13 \mathrm{q} 13$ in patients with MGUS and multiple myeloma. Exp Hematol 2009; 37: 234-244. doi:10.1016/j.exphem.2008.10.014

29. Yip S, Iafrate AJ, Louis DN. Molecular Diagnostic Testing in Malignant Gliomas: A Practical Update on Predictive Markers. J Neuropathol Exp Neurol 2008; 67: 1-15. doi:10.1097/nen.0b013e31815f65fb

30. Hesson LB, Krex D, Latif F. Epigenetic markers in human gliomas: prospects for therapeutic intervention. $E x$ pert Rev Neurother 2008, 8, 1475-1496. doi:10.158 $\underline{6 / 14737175.8 .10 .1475}$

31. Pc B, Schthauer BW, Vogel FS. Surgical Pathology of the Nervous System and its coverings. Philadelphia: churchill Livingstone, 2002.

Received 10 November, 2009; accepted 6 December, 2009; published online 9 December, 2009.

Copyright: (c) 2009 Chenchen Bao et al. This is an openaccess article distributed under the terms of the Creative Commons Attribution License, which permits unrestricted use, distribution, and reproduction in any medium, provided the original author and source are credited 